\title{
Genesis of the Floquet Hofstadter butterfly
}

\author{
S. H. Kooi, ${ }^{1}$ A. Quelle, ${ }^{1}$ W. Beugeling, ${ }^{2}$ and C. Morais Smith ${ }^{1}$ \\ ${ }^{1}$ Institute for Theoretical Physics, Center for Extreme Matter and Emergent Phenomena, Utrecht University, \\ Princetonplein 5, 3584 CC Utrecht, The Netherlands \\ ${ }^{2}$ Physikalisches Institut, Universität Würzburg, Am Hubland, 97074 Würzburg, Germany
}

(Received 20 March 2018; revised manuscript received 9 August 2018; published 14 September 2018)

\begin{abstract}
We investigate theoretically the spectrum of a graphenelike sample (honeycomb lattice) subjected to a perpendicular magnetic field and irradiated by circularly polarized light. This system is studied using the Floquet formalism, and the resulting Hofstadter spectrum is analyzed for different regimes of the driving frequency. For lower frequencies, resonances of various copies of the spectrum lead to intricate formations of topological gaps. In the Landau-level regime, new winglike gaps emerge upon reducing the driving frequency, thus revealing the possibility of dynamically tuning the formation of the Hofstadter butterfly. In this regime, an effective model may be analytically derived, which allows us to retrace the energy levels that exhibit avoided crossings and ultimately lead to gap structures with a winglike shape. At high frequencies, we find that gaps open for various fluxes at $E=0$, and upon increasing the amplitude of the driving, gaps also close and reopen at other energies. The topological invariants of these gaps are calculated and the resulting spectrum is elucidated. We suggest opportunities for experimental realization and discuss similarities with Landau-level structures in nondriven systems.
\end{abstract}

DOI: 10.1103/PhysRevB.98.115124

\section{INTRODUCTION}

The complex fractal structure of the Hofstadter butterfly, which reveals the interplay between the lattice constant and the magnetic length when a perpendicular magnetic field is applied to a crystal lattice, has fascinated researchers since its first theoretical prediction [1]. However, its experimental realization seemed to be impossible at first sight, because for typical crystal lattice spacings, the magnetic field required to observe the butterfly is of the order of thousands of tesla. Recently, moiré superlattices, obtained when depositing graphene on mismatched substrates, such as hBN, have been realized $[2,3]$. These structures have an effective lattice spacing that is an order of magnitude larger than the usual crystal lattices. This has brought the required magnetic-field strength within experimental reach, and enabled the observation of the Hofstadter butterfly spectra [4,5]. In addition, the Hofstadter butterfly has been proposed in nanophotonic devices [6] and for bosons in optical lattices [7-9], where it has also been experimentally realized $[10,11]$.

All these studies were done in equilibrium, and so far out-of-equilibrium Hofstadter setups have not received much attention, although driven systems have been under intense scrutiny recently [12-27]. In particular, time-periodic driving attracted great interest because it can be conveniently described in the framework of Floquet theory [12,14,28,29]. This allows one to define quasistatic properties of the driven system that can be measured and is a tuning knob for quantum simulations both in condensed-matter and cold-atom experiments. The quasienergy spectrum obtained using Floquet theory is periodic, with a period proportional to the driving frequency. Recently, periodically driven systems have been observed in photonics [20], condensed-matter [21], and cold-atom experiments $[22,30]$.
Periodic driving described by Floquet theory can lead to many interesting topological phase transitions [13-18,31], characterized by a slightly different topological invariant than for the undriven case [32-35]. For example, Floquet theory predicts additional topological phases in the Kitaev chain [19]. Topological behavior induced by periodic driving has been observed experimentally in photonic waveguides [20], and a gap opening has been detected on the surface of a topological insulator upon irradiation with circularly polarized light [21]. The Berry curvature of such Floquet Bloch bands has also been explicitly measured [22].

Several recent works have been dedicated to the investigation of the driven Hofstadter model. In Refs. [25,26], the driven Hofstadter model has been investigated on a square lattice for a specific flux $\phi=1 / 3$ (in units of the flux quantum $\phi_{0}=h / e$ ), and for two different driving protocols. In both cases, the authors find counter-propagating edge modes in the quasienergy spectrum, crossing $E= \pm \pi \hbar / T$ (where $T$ is the period of the driving). The Hofstadter butterfly for a driven honeycomb lattice has been studied in Ref. [36], with an extensive Chern number analysis. In Ref. [27], a transition from the half-integer to the integer quantum Hall effect has been theoretically proposed to occur upon elliptical driving of an ac field.

Here, we show that the Floquet method can be used to unveil the formation of the Hofstadter butterfly at low magnetic fields by adding a periodic driving. Upon tuning the frequency, the bands start to overlap and avoided crossings occur, that lead to the formation of wings. At small flux, where the spectrum has a Landau-level structure, the procedure can be analytically monitored using the Floquet formalism. In doing so, we gain insight on the mechanism of hybridization between Landau levels. For larger magnetic fields, we perform numerical calculations to obtain the full butterfly spectrum for various frequencies. 
The outline of this paper is as follows. In Sec. II, we introduce the model and explain the details of Floquet theory. In Sec. III, we present numerical results for the small-flux regime and derive an effective model to explain the mixing of the Landau levels. In Sec. IV, we present and analyze our numerical results for the full range of flux, in both highand low-frequency regimes. Our findings are summarized in Sec. V.

\section{THE MODEL}

We consider a honeycomb lattice (e.g., a graphene monolayer) subject to a perpendicular magnetic field and to irradiation by circularly polarized light [see Fig. 1(a)]. The system is described by a tight-binding model of electrons on a honeycomb lattice, where the background magnetic field and the circularly polarized light are included through a vector potential A, via Peierls substitution. The Hamiltonian reads

$$
H=-J \sum_{l=1,2,3} \sum_{\mathbf{r}}\left|\mathbf{r}+\delta_{l}\right\rangle e^{i \int d \mathbf{s} \cdot \mathbf{A}}\langle\mathbf{r}|+\text { H.c. }
$$

where $J$ is the hopping parameter, $\mathbf{r}$ is the position of a site, $\delta_{l}$ are the nearest-neighbor vectors of the honeycomb lattice, and $d \mathbf{s}$ parametrizes the path between two sites, $\mathbf{r}$ and $\mathbf{r}+\delta_{l}$. The vector potential consists of two contributions,

$$
\mathbf{A}(\mathbf{r}, t)=\mathbf{A}_{\mathrm{mag}}(\mathbf{r})+\mathbf{A}_{\text {light }}(t) .
$$

The first term is due to the background magnetic field, which will be described in the Landau gauge,

$$
\mathbf{A}_{\text {mag }}(\mathbf{r})=-\frac{e}{\hbar}(B y, 0),
$$

(a)
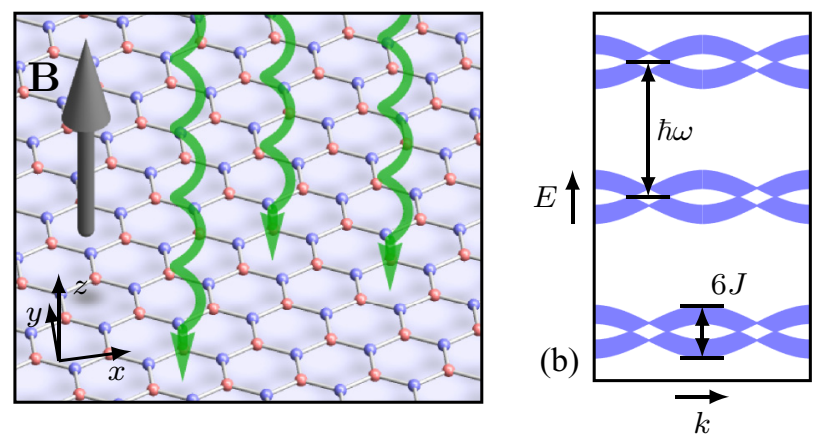

FIG. 1. (a) Schematic setup of our model. The honeycomb lattice is subjected to a perpendicular magnetic field, and is simultaneously irradiated by circularly polarized light. (b) Quasienergy spectrum of the Floquet model. The generic feature of the Floquet spectrum is the periodicity with $\hbar \omega$ in the vertical direction.

where $e$ is the electron charge and $\hbar$ the reduced Planck constant. The second contribution comes from the circularly polarized light and is periodic in time,

$$
\mathbf{A}_{\text {light }}(t)=A_{0}(-\sin (\omega t), \cos (\omega t)),
$$

where $A_{0}$ is the amplitude, $\omega$ is the frequency of the light and $t$ denotes time.

Let us start by considering the static Hamiltonian, with $\mathbf{A}_{\text {light }}=0$. A Fourier transformation then yields the Harper equation of the honeycomb lattice (we set the lattice spacing to unity):

$$
\begin{aligned}
-E\left(\begin{array}{ll}
1 & 0 \\
0 & 1
\end{array}\right) \psi_{r}(k)= & J\left(\begin{array}{cc}
0 & 1 \\
1 & 0
\end{array}\right) \psi_{r}(k)+J e^{i \frac{\sqrt{3}}{2} k_{x}}\left(\begin{array}{cc}
0 & e^{\frac{3}{2} i\left(k_{y}+\frac{2}{3} \pi \frac{r p}{q}\right)} \\
e^{-\frac{3}{2} i\left(k_{y}+\frac{2}{3} \pi \frac{r p}{q}\right)} & 0
\end{array}\right) \psi_{r+1}(k) \\
& +J e^{-i \frac{\sqrt{3}}{2} k_{x}}\left(\begin{array}{cc}
0 & e^{\frac{3}{2} i\left(k_{y}+\frac{2}{3} \pi \frac{r p}{q}\right)} \\
e^{-\frac{3}{2} i\left(k_{y}+\frac{2}{3} \pi \frac{r p}{q}\right)} & 0
\end{array}\right) \psi_{r-1}(k),
\end{aligned}
$$

where $r=1, \ldots, q$, and $\psi_{q+1}=\psi_{1}$, with

$$
\psi_{r}(k)=\left(\psi_{r, k_{x}, k_{y}}^{\mathrm{A}}, \psi_{r, k_{x}, k_{y}}^{\mathrm{B}}\right) .
$$

Here, the components $\psi^{\mathrm{A}}$ and $\psi^{\mathrm{B}}$ refer to the two sublattices of the honeycomb lattice, and we have taken the flux per unit cell to be $\phi=p / q$ in units of the flux quantum $\phi_{0}$, where $p$ and $q$ are coprime integers. Thus, the matrices in the Harper equation [Eq. (5)] act in sublattice space.

To describe the influence of the circularly polarized light, we now also consider $\mathbf{A}_{\text {light }}$. This will amount to each hopping picking up a phase,

$$
e^{i \int d \mathbf{s} \cdot \mathbf{A}_{\text {light }}}=\exp \left\{i A_{0}[-\cos (\theta) \sin (\omega t)+\sin (\theta) \cos (\omega t)]\right\},
$$

where $\theta$ is the angle between the bond and the $x$ axis. Since the Hamiltonian is now periodic in time, we can define the Floquet
Hamiltonian by [12]

$$
H_{\text {Floq }}=\frac{i \hbar}{T} \ln [U(T, 0)] .
$$

Here, $T=2 \pi / \omega$ is the period of the driving and $U(T, 0)$ is the time-evolution operator, which may be found by numerically solving the Schrödinger equation

$$
i \hbar \frac{\partial U\left(t, t^{\prime}\right)}{\partial t}=H(t) U\left(t, t^{\prime}\right) .
$$

By calculating the eigenvalues and eigenstates of $H_{\text {Floq }}$, we can determine the quasi-static behavior of the system at stroboscopic timescales larger than $T$. The time-periodic Hamiltonian can thus be expanded into the Fourier coefficients $H_{n}$, as

$$
H(t)=\sum_{n} H_{n} e^{i n \omega t}
$$


The eigenenergies of the Floquet Hamiltonian then follow from diagonalization of

$$
H_{\text {Floq }}=\left(\begin{array}{ccccc}
\ddots & \vdots & \vdots & \vdots & . \cdot \\
\cdots & H_{0}+\hbar \omega & H_{1} & H_{2} & \cdots \\
\cdots & H_{-1} & H_{0} & H_{1} & \cdots \\
\cdots & H_{-2} & H_{-1} & H_{0}-\hbar \omega & \cdots \\
. \cdot & \vdots & \vdots & \vdots & \ddots
\end{array}\right) .
$$

We can interpret the Hamiltonian Eq. (11) as an infinite set of copies of the Hamiltonian $H_{0}$, separated by energies $\hbar \omega$, as illustrated by Fig. 1(b). These copies are then mixed by the offdiagonal elements. If $\hbar \omega$ is much larger than the bandwidth $6 J$ of the spectrum of $H_{0}$, this mixing will be negligible. However, when $\hbar \omega$ becomes comparable to $6 J$, the different copies of $H_{0}$ start to overlap and the mixing terms become important.

For high frequencies, the Floquet Hamiltonian can be expanded to first nontrivial order as [12,31,37-40]

$$
H_{\text {Floq }} \approx H_{0}+\left[H_{-1}, H_{1}\right] / \hbar \omega .
$$

\section{LANDAU-LEVEL REGIME}

We will first focus our attention to the small-flux limit, where the Hofstadter spectrum typically exhibits a Landaulevel structure. In Fig. 2, we plot the energies as a function of the flux $\phi$ for different values of the driving frequency $\omega$. We consider the regime where the frequency becomes comparable
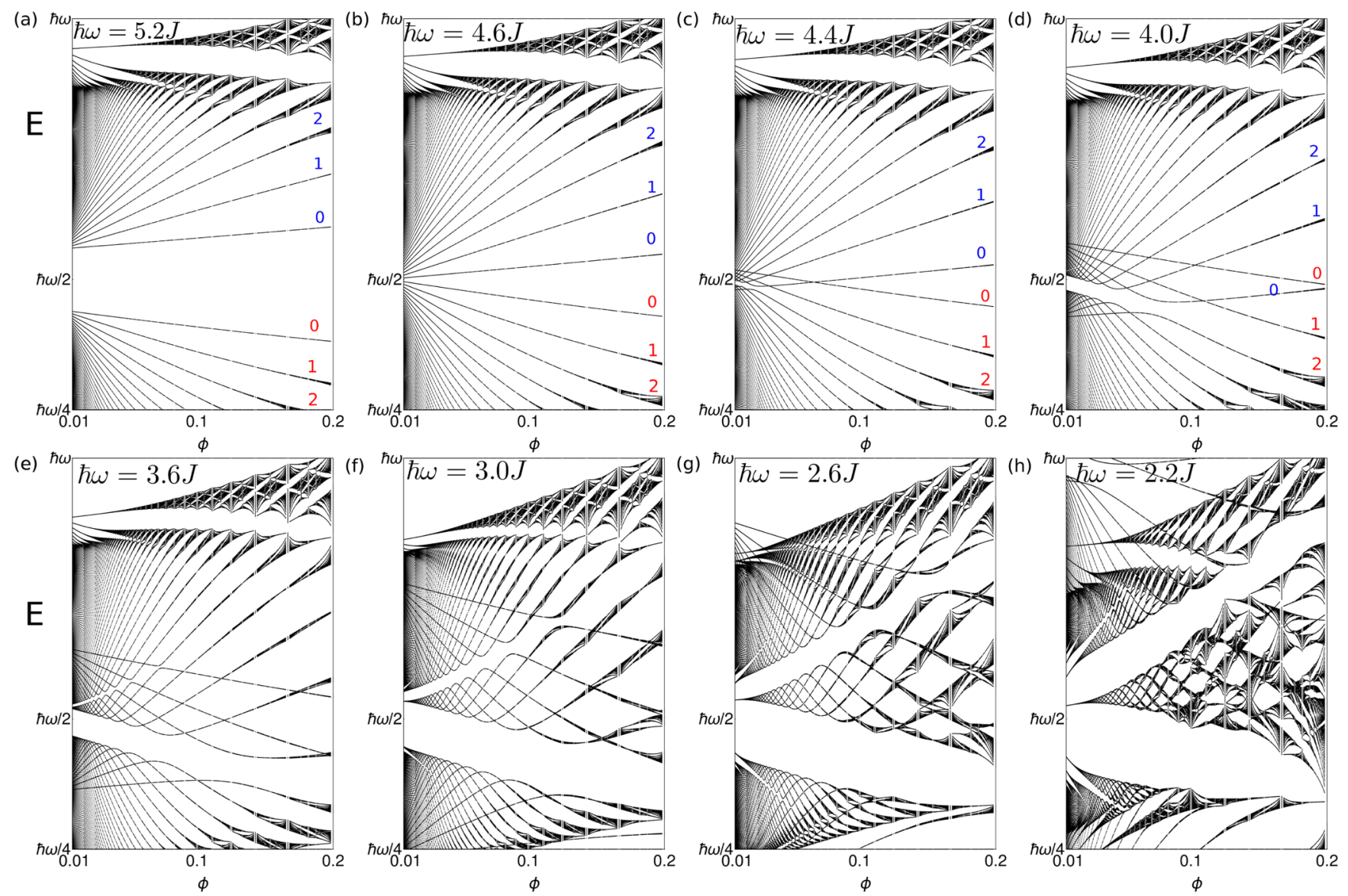

to the bandwidth $6 J$. In Fig. 2(a), for $\hbar \omega=5.2 J$, we observe that two subsequent copies of $H_{0}$ are still well separated. (The two copies shown here live in the intervals $[-\hbar \omega / 2, \hbar \omega / 2]$ and $[\hbar \omega / 2,3 \hbar \omega / 2]$, respectively.) The coupling between the two copies reduces their width to a value smaller than $6 \mathrm{~J}$. Upon lowering the frequency, the two copies of bands come closer to each other and start to overlap. We see this process in Figs. 2(c)-2(h). In Figs. 2(b) and 2(c), the initial overlap of the bands takes place. Curiously, the top two Landau levels of the lower copy do not mix with any Landau level of the upper copy, while the rest hybridizes and a gap opens due to their avoided crossings. We will explain this behavior in the next section using an effective model to describe this regime. In Figs. 2(e)-2(h), one sees additional gaps opening, and one observes that the two largest gaps acquire a shape that resembles the wings of the undriven Hofstadter butterfly.

\section{Effective model}

We now derive an effective model to describe the initial overlap of the two copies of bands displayed in Figs. 2(b)-2(d), aiming at understanding why the top two bands of the lower copy do not hybridize with the bands of the upper copy. To do so, we zoom in around $E \approx \hbar \omega / 2$, where the overlap occurs. Our starting point is the Hamiltonian in Eq. (11). Since we are interested in the regime where two copies start overlapping, $\hbar \omega \lesssim 6 J$, at energy $E \approx \hbar \omega / 2$, we can restrict ourselves to

FIG. 2. Spectra for small fluxes $\phi$, in the Landau-level regime, plotted for various values of $\omega$, with $A_{0}=1$. In the first four plots we label the first three Landau levels of the upper (blue) and lower (red) copy. 
two copies of $H_{0}$. Here, we take the ones centered at $E=0$ and $E=\hbar \omega$, and consider their mixing, of which the dominant contribution stems from $H_{1}$ and $H_{-1}$. The mixing with levels in more distant Floquet copies is negligible, as the effect is suppressed with increasing energy difference. (A similar analysis is done in Ref. [35].) The effective Hamiltonian then becomes

$$
H_{\text {Floq }}^{\text {eff }}=\left(\begin{array}{cc}
H_{0}+\hbar \omega & H_{1} \\
H_{-1} & H_{0}
\end{array}\right) .
$$

To derive analytical expressions for $H_{n}$, with $n=-1,0,1$, we initially solve the problem at zero dc magnetic field $(\phi=0)$, including only the time-dependent perturbation. In this case, $H_{n}$ can be obtained by making the following substitution in the Hamiltonian,

$$
\begin{aligned}
J \rightarrow & \frac{J}{T} \int_{0}^{T} d t^{\prime} \exp \left\{i A _ { 0 } \left[-\cos (\theta) \sin \left(\omega t^{\prime}\right)\right.\right. \\
& \left.\left.+\sin (\theta) \cos \left(\omega t^{\prime}\right)\right]\right\} \exp \left\{i n \omega t^{\prime}\right\} \\
= & J J_{n}\left(A_{0}\right) \exp (i n \theta),
\end{aligned}
$$

where $J_{n}$ is the $n$th Bessel function of the first kind. Applying this substitution to the tight-binding Hamiltonian [Eq. (1)] of the honeycomb lattice, we obtain

$$
H_{n}=\left(\begin{array}{cc}
0 & h_{n} \\
h_{n}^{\prime} & 0
\end{array}\right),
$$

where

$$
\begin{aligned}
h_{n}= & -J\left[e^{i\left(k_{y} \frac{1}{2}+k_{x} \frac{\sqrt{3}}{2}\right)} e^{i n \frac{5}{6} \pi}+e^{i\left(k_{y} \frac{1}{2}-k_{x} \frac{\sqrt{3}}{2}\right)} e^{i n \frac{\pi}{6}}\right. \\
& \left.+e^{-i k_{y}} e^{i n \pi \frac{3}{2}}\right] J_{n}\left(A_{0}\right), \\
h_{n}^{\prime}= & -J\left[e^{-i\left(k_{y} \frac{1}{2}+k_{x} \frac{\sqrt{3}}{2}\right)} e^{-i n \frac{\pi}{6}}+e^{-i\left(k_{y} \frac{1}{2}-k_{x} \frac{\sqrt{3}}{2}\right)} e^{-i n \frac{5}{6} \pi}\right. \\
+ & \left.e^{i k_{y}} e^{i n \frac{\pi}{2}}\right] J_{n}\left(A_{0}\right) .
\end{aligned}
$$

At small $\phi$, we enter the Landau-level regime. Because of the suppression of the mixing with energy difference, the strongest overlap occurs between the highest and lowest Landau levels of two neighboring Floquet copies. This observation justifies an expansion of the Hamiltonian around the maximum of the spectrum at $\mathbf{k}=0$. The dispersion is quadratic in leading order, and we find

$$
H_{0}=-3 J J_{0}\left(A_{0}\right)\left[1-\frac{1}{4}\left(k_{x}^{2}+k_{y}^{2}\right)\right] \sigma_{x},
$$

where $\sigma_{x}$ is a Pauli matrix in the sublattice pseudospin space, and we have omitted the higher-order terms. We now introduce the magnetic field by minimal Peierls substitution, and then the standard ladder operators $a$ and $a^{\dagger}$ to find

$$
H_{0}=-3 J J_{0}\left(A_{0}\right)\left[1-\frac{1}{2 l_{B}^{2}}\left(a^{\dagger} a+\frac{1}{2}\right)\right] \sigma_{x},
$$

where $l_{B}=\sqrt{\hbar / e B}$ is the magnetic length in terms of the magnetic field $B$. (We recall that the lattice spacing has been set to unity.) The term $H_{1}$, mixing two copies of the butterfly spectrum, is obtained by a similar calculation,

$$
H_{1}=J J_{1}\left(A_{0}\right) \frac{3}{2}\left[\frac{\sqrt{2}}{l_{B}} a^{\dagger} \sigma_{x}+\frac{1}{2 l_{B}^{2}} a a \sigma_{y}\right] .
$$

The eigenstates $\psi_{n, \pm}$ of $H_{0}$ have the same structure as the eigenstates of $\sigma_{x}$,

$$
\psi_{n, \pm}=\frac{1}{\sqrt{2}}\left(\begin{array}{c}
|n\rangle \\
\mp|n\rangle
\end{array}\right)
$$

and their energies are

$$
E_{n, \pm}= \pm 3 J J_{0}\left(A_{0}\right)\left[1-\frac{1}{2 l_{B}^{2}}\left(n+\frac{1}{2}\right)\right] .
$$

These results are compatible with Ref. [35], which discusses the zero-field case.

One observes that for each Floquet copy, which we label by $r$ in the following, there are two sequences of Landau levels: one where the zeroth Landau level is at the top of the spectrum, and one where it is at the bottom of the spectrum, labeled by + and - , respectively. In $H_{1}$, the term proportional to $\sigma_{y}$ couples $\psi_{n,+, r}$ with $\psi_{n^{\prime},-, r+1}$ and $\psi_{n,-, r}$ with $\psi_{n^{\prime},+, r+1}$. The former pair constitutes states very close in energy (energy difference $\Delta E \ll \hbar \omega)$, whereas the latter pair are distant states $(\Delta E \approx 2 \hbar \omega)$. The term proportional to $\sigma_{x}$ couples $\psi_{n, \pm, r}$ with $\psi_{n^{\prime}, \pm, r+1}$, whose energy difference is $\Delta E \approx \hbar \omega$. From perturbation theory, it follows that the energy shift due to the mixing term scales as $1 / \Delta E$. Consequently, hybridization between the states $\psi_{n,+, r}$ and $\psi_{n^{\prime},-, r+1}$ is significant, whereas the couplings between the other pairs have negligible effects.

The strong mixing between $\psi_{n,+, r}$ and $\psi_{n^{\prime},-, r+1}$ is due to the matrix element proportional to $a a$ in Eq. (20). Thus, hybridization occurs between these states if $n^{\prime}=n-2$. In Figs. 2(c) and 2(d), we indeed observe that avoided crossings occur between the Landau levels labeled 0 (blue) and 2 (red), between 1 (blue) and 3 (red), etc. The top two Landau levels of the lower copy $(n=0,1$, labeled in red) do not have a partner; they do not hybridize with any of the bands of the upper copy (labeled in blue).

\section{NUMERICAL RESULTS}

We now go beyond the low-flux regime and study the full Hofstadter butterfly. We present numerical results for both high frequencies, when the periodicity of the spectrum is much larger than the bandwidth, and lower frequencies, where overlaps are observed.

\section{A. High-frequency regime}

In Fig. 3, we plot the energy levels as a function of the flux per plaquette $\phi$, for several values of the amplitude at a frequency of $\omega=12 J / \hbar$, such that the periodicity of the spectrum $\hbar \omega$ is larger than the bandwidth $\sim 6 J$. Thus, there are only resonances within one Floquet copy of the spectrum.

The colors of the gaps correspond to the associated topological invariants, which are obtained by using the Středa formula [41],

$$
\sigma_{\mathrm{H}}=2 \frac{e^{2}}{h} \frac{\partial N}{\partial \phi},
$$

which provides the Hall conductivity $\sigma_{\mathrm{H}}$, in terms of the integrated density of states $N$ and the conductance quantum $e^{2} / h$. We have checked and confirmed that the resulting values of $\sigma_{\mathrm{H}}$ from Eq. (23) are identical to those obtained by counting the 

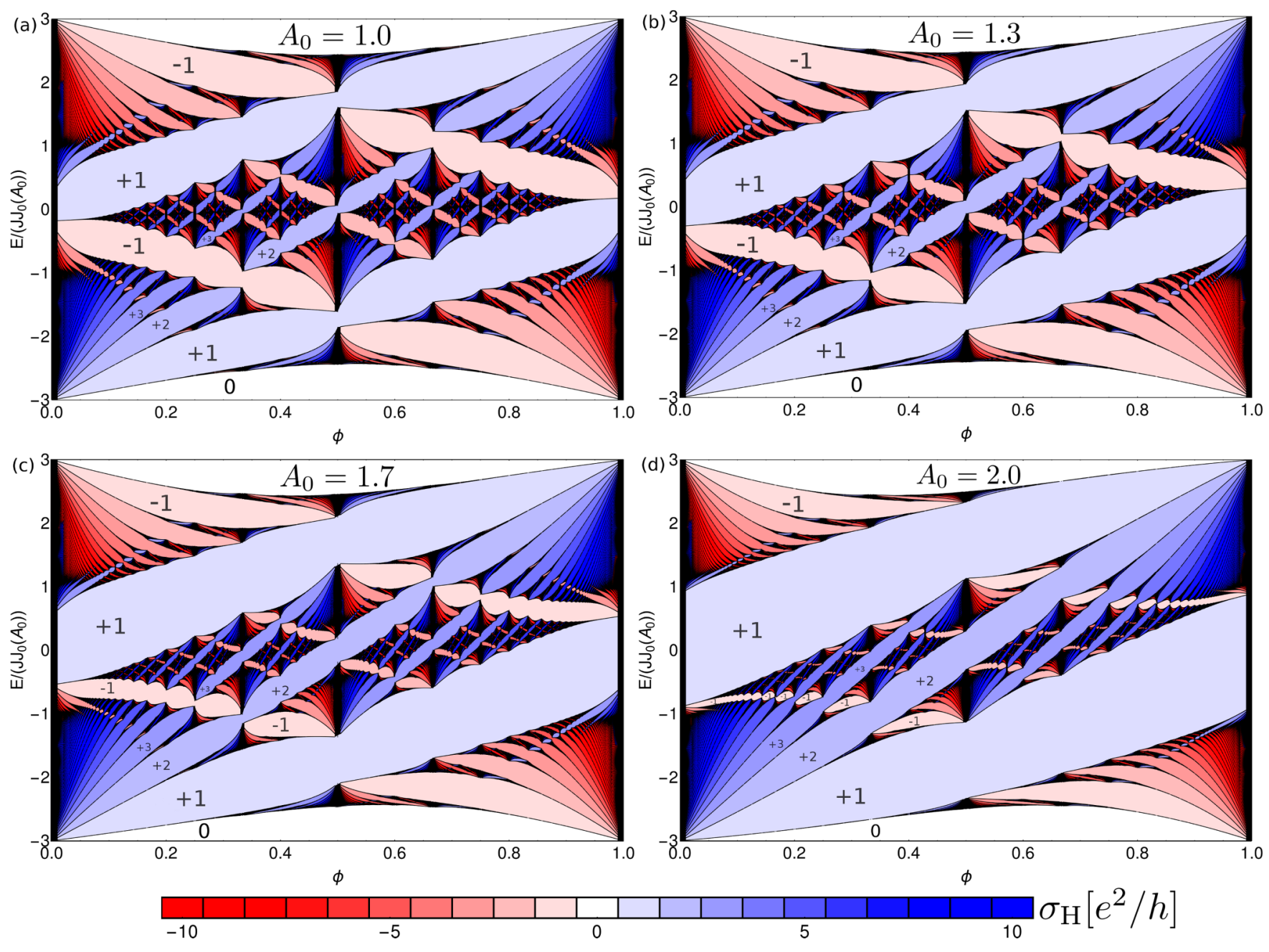

FIG. 3. Full spectrum plotted for $\hbar \omega=12 \mathrm{~J}$ and various values of $A_{0}$. The colors of the gaps correspond to the number of left (red) or right (blue) moving edge states.

number of chiral edge states in a ribbon-geometry calculation of the dispersion. Identical results can be obtained from explicit calculation of the Chern numbers [36,42], however at a higher computational expense. Although the topological invariant of Floquet systems is not the same as for static systems [32], in the high-frequency regime the Středa formula still yields the correct conductivity values because there is still a trivial gap between different copies of the original spectrum.

For $\phi=0$ (no magnetic field), circularly polarized light opens up a topological gap in the honeycomb system, and realizes a dynamical Haldane model [16,30,43-45]. Since the spectrum is continuous as a function of $\phi$, this gap must persist for non-zero $\phi$. From Fig. 3, we see indeed that it connects with the large gap above $E=0$, which also has topological invariant +1 . If we create a gap with opposite winding number (by reversing the polarization of the light), the gap that opens up at $\phi=0$ would connect to the lower large gap with invariant -1 . At other fractional fluxes, such as $\phi=1 / 2,1 / 3,1 / 4$, non-trivial gaps also open at $E=0$ with the same chirality and with the topological invariant equal to the denominator of the rational flux.

As we increase the amplitude of the light $A_{0}$, we change the effective couplings $J J_{n}\left(A_{0}\right)$ [see Eq. (14)] in the Floquet
Hamiltonian, which induces additional topological phase transitions. These will happen by the closing and opening of a gap that already exists without driving [46]. An example can be observed at $\phi=1 / 3$, where the large gap around $E \approx-1$ with invariant -1 becomes smaller for $A_{0}=1.3$, closes around $A_{0} \approx 1.5$ and is reopened at $A_{0}=1.7$.

The gap closing occurs at three points in the Brillouin zone and the topological invariant changes from -1 to +2 (see colors in Fig. 3), consistent with the number of gap closing points. Because the system still has magnetic-translation symmetry, the topological invariant must satisfy the Diophantine equation $[25,47]$

$$
p c+q d=1,
$$

for flux $\phi=p / q$, where $c$ is the topological invariant and $d$ is integer. This means that the topological invariant can only change in multiples of $q$, which indeed agrees with our observation at the third gap for flux $\phi=1 / 3$.

\section{B. Higher photon resonances}

As we lower $\omega$, and $\hbar \omega$ becomes comparable to the bandwidth $(\lesssim 6 J)$, bands from the next copy will start interacting 
(a) $\hbar w$

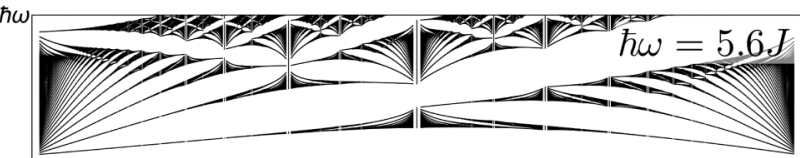

$\hbar \omega / 2$
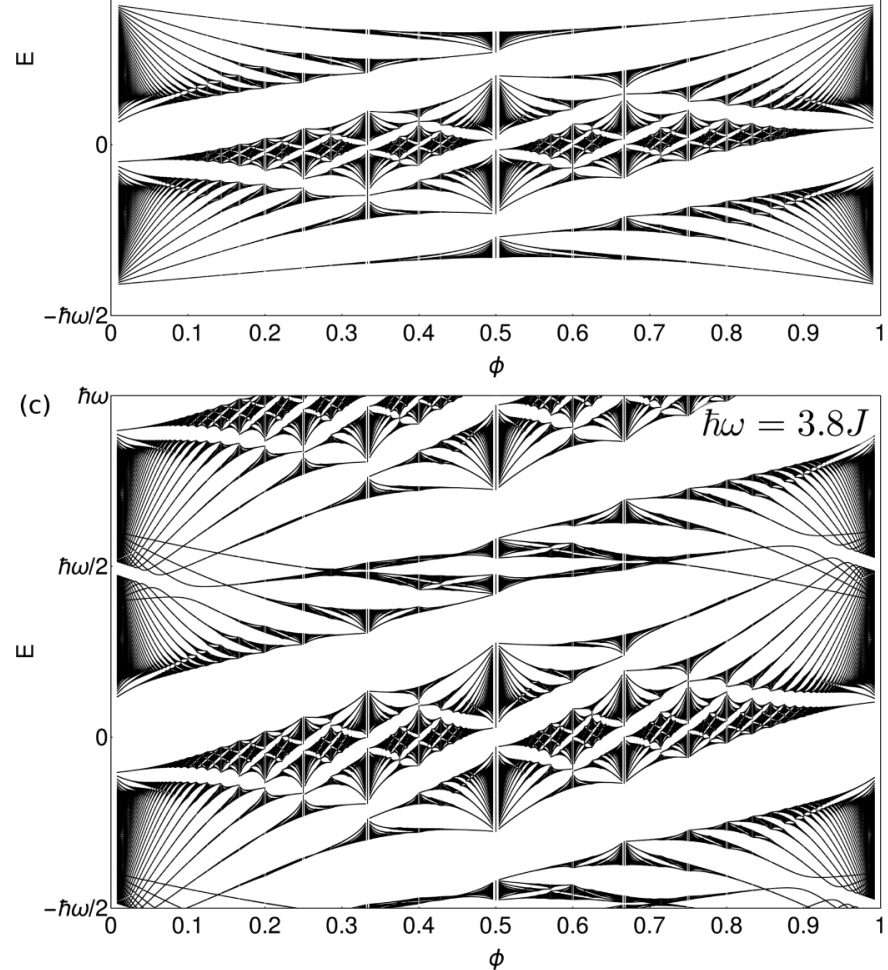

(e) $\hbar \omega$

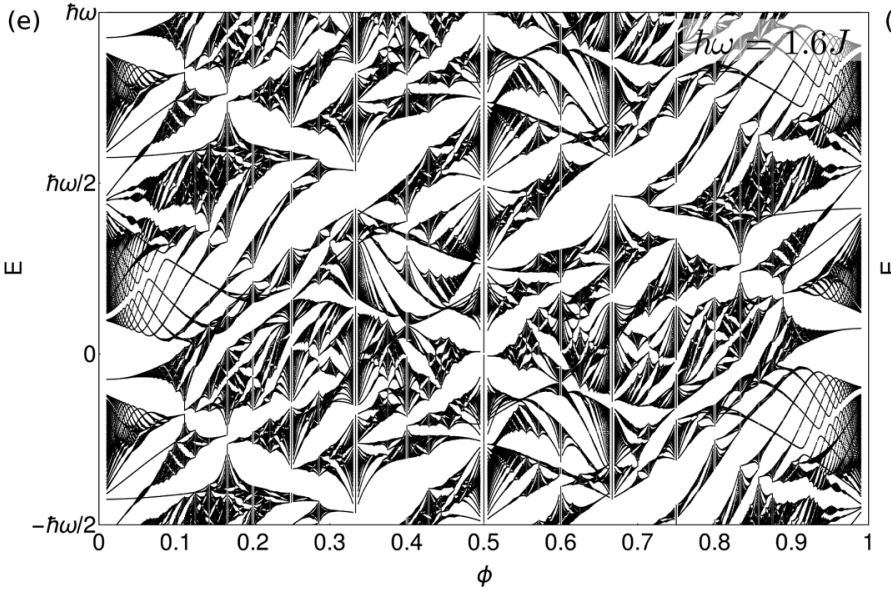

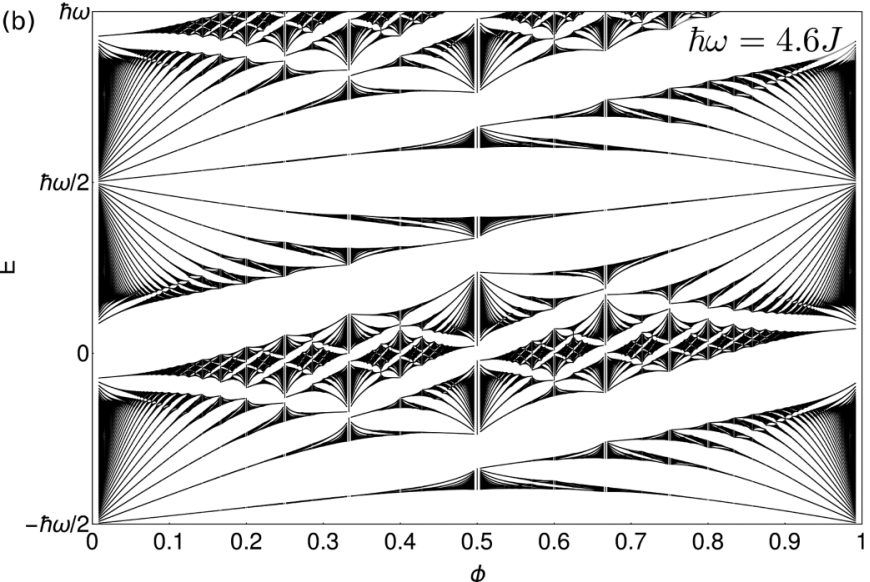

(d)
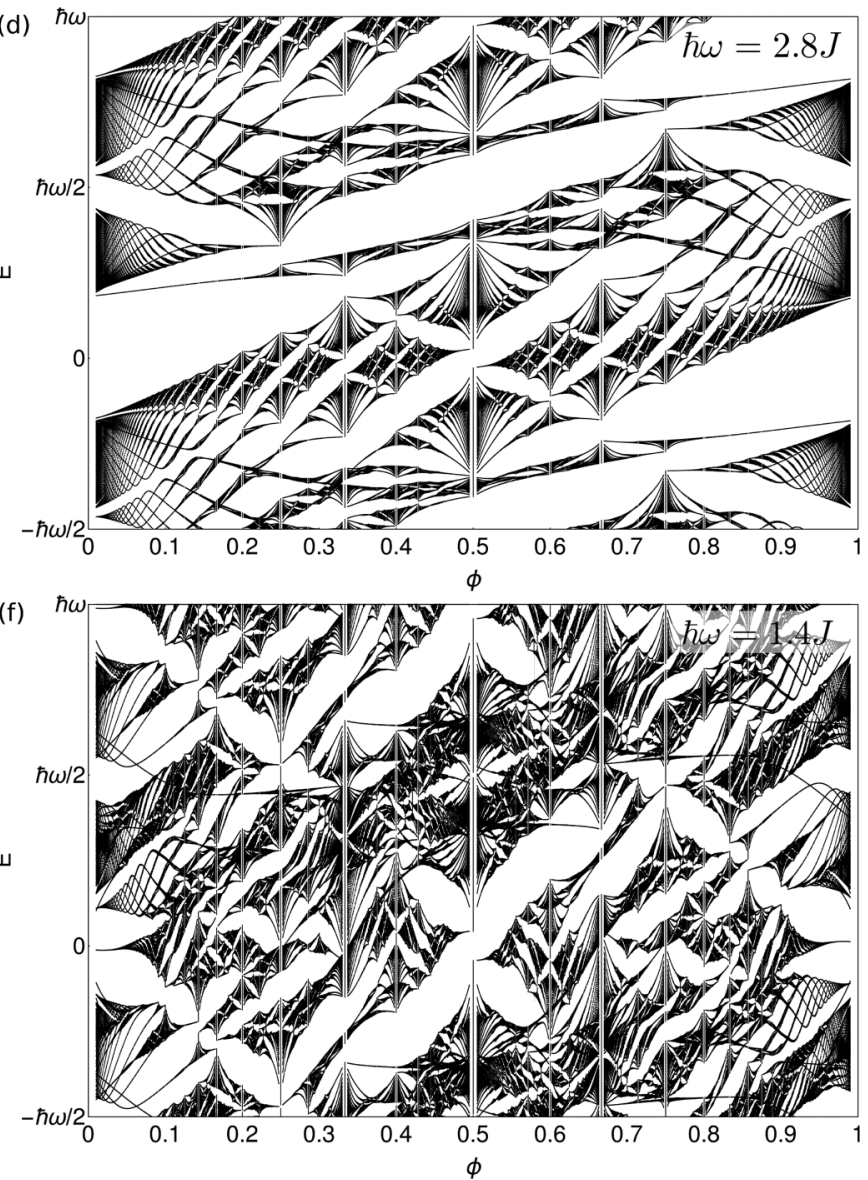

FIG. 4. Full spectra plotted for various values of the frequency and $A_{0}=1$.

with each other (this regime in the case of $\phi=0$ has been studied in Ref. [31]). We plot the spectrum for $A_{0}=1$ and various frequencies in Fig. 4. In Fig. 4(a) $(\hbar \omega=5.6 J)$ there is still a gap between the first and second copy of bands. In Fig. 4(b), the bands are at the verge of crossing, and in Fig. 4(c) $(\hbar \omega \ll 6 J)$ there is an overlap between the two copies. The mixing of the energy bands gives rise to an intricate spectrum, and also causes many topological phase transitions. One example is the gap that appears at $E \approx \hbar \omega / 2$ around $\phi=1 / 2$ in Fig. 4(c). Since the different copies are now starting to overlap, the periodicity of the spectrum makes it difficult to define a reference value for the filling (integrated density of states $N$ ) and the Středa formula no longer a priori provides the correct topological invariant. As we decrease $\omega$ even further, an almost flat band appears for small $\phi$ [see Fig. 4(d)], where the gap below (above) has Hall conductivity $+1(+2)$. In this regime, it is possible to clearly distinguish between two gaps with a different number of edge states (the gap above has two, the gap below one), where the gap above the flat state has been created by the Floquet driving. This could facilitate experiments, since the narrow and flat band persists for a wide range of flux. 


\section{CONCLUSION}

By irradiating a honeycomb lattice subjected to a perpendicular magnetic field with circularly polarized light, its Hofstadter butterfly exhibits an even richer structure than its static counterpart. In particular, we can follow the formation of winglike structures in the spectrum at low flux and low frequencies. The highest two Landau levels of the spectrum do not mix with the overlapping copy, while the other levels do, as captured by our effective analytical model.

To realize these features experimentally, the Floquet perturbation and the flux per unit cell need to be large. The Floquet perturbations enter through Bessel functions as factors of (reintroducing the lattice constant $c$ ) $J_{n}\left(A_{0} c\right)$, which shows that a larger lattice constant would increase the Floquet strength as well as the flux per unit cell. This makes honeycomb structures with large lattice constants a natural place to realize this system. Such structures can be for example lattices of nanocrystals [48,49] or optical lattices [50]. In optical lattices, one can also implement shaking protocols [15,51]. A circular shaking protocol will induce a vector potential of the same form as Eq. (4) [35,52]. The amplitude, however, will grow linearly with the frequency $\omega$, while for light $A_{0} \sim e E / \hbar \omega$. As the required frequencies are quite large, this will aid in an experimental realization. In such a setup, $\hbar \omega \approx 2.7 \mathrm{~J}$ can be realized, which would be sufficient to observe the newly formed wings.

The structures observed at the process of opening the wings are reminiscent of generic hybridized dispersions. For example, in semiconductor quantum wells (e.g., $\mathrm{HgCdTe} / \mathrm{HgTe}$ ) $[29,53,54]$, gaps open between Landau levels in the valence band. In that case, the "warping terms," which make the dispersion nonisotropic, induce a coupling between Landau levels with indices $n$ and $n \pm 4$. The mechanism for the formation of these gaps is thus analogous to the one governing the wing formation in the present Floquet model. This analogy suggests a potential application of Floquet systems as simulator of band structures of generic condensed matter systems. In particular, such simulations could provide more insight into hybridization in complicated Landau-level spectra.

\section{ACKNOWLEDGMENTS}

We thank G. Platero for useful discussions. The work by A.Q. and C.M.S. is part of the D-ITP consortium, a program of the Netherlands Organisation for Scientific Research (NWO) that is funded by the Dutch Ministry of Education, Culture, and Science (OCW). S.K. acknowledges support from a NWOGraduate Program grant.
[1] D. R. Hofstadter, Phys. Rev. B 14, 2239 (1976).

[2] M. Yankowitz, J. Xue, D. Cormode, J. D. Sanchez-Yamagishi, K. Watanabe, T. Taniguchi, P. Jarillo-Herrero, P. Jacquod, and B. J. LeRoy, Nat. Phys. 8, 382 (2012).

[3] J. Xue, J. Sanchez-Yamagishi, D. Bulmash, P. Jacquod, A. Deshpande, K. Watanabe, T. Taniguchi, P. Jarillo-Herrero, and B. J. LeRoy, Nat. Mater. 10, 282 (2011).

[4] L. Ponomarenko, R. Gorbachev, G. Yu, D. Elias, R. Jalil, A. Patel, A. Mishchenko, A. Mayorov, C. Woods, J. Wallbank et al., Nature 497, 594 (2013).

[5] C. Dean, L. Wang, P. Maher, C. Forsythe, F. Ghahari, Y. Gao, J. Katoch, M. Ishigami, P. Moon, M. Koshino et al., Nature 497, 598 (2013).

[6] M. Hafezi, E. A. Demler, M. D. Lukin, and J. M. Taylor, Nat. Phys. 7, 907 (2011).

[7] D. Jaksch and P. Zoller, New J. Phys. 5, 56 (2003).

[8] J. Dalibard, F. Gerbier, G. Juzeliūnas, and P. Öhberg, Rev. Mod. Phys. 83, 1523 (2011).

[9] N. Goldman, G. Juzeliūnas, P. Öhberg, and I. B. Spielman, Rep. Prog. Phys. 77, 126401 (2014).

[10] H. Miyake, G. A. Siviloglou, C. J. Kennedy, W. C. Burton, and W. Ketterle, Phys. Rev. Lett. 111, 185302 (2013).

[11] M. Aidelsburger, M. Atala, M. Lohse, J. T. Barreiro, B. Paredes, and I. Bloch, Phys. Rev. Lett. 111, 185301 (2013).

[12] A. Eckardt, Rev. Mod. Phys. 89, 011004 (2017).

[13] P. Titum, E. Berg, M. S. Rudner, G. Refael, and N. H. Lindner, Phys. Rev. X 6, 021013 (2016).

[14] N. H. Lindner, G. Refael, and V. Galitski, Nat. Phys. 7, 490 (2011).

[15] W. Zheng and H. Zhai, Phys. Rev. A 89, 061603 (2014).

[16] A. Quelle and C. Morais Smith, Phys. Rev. B 90, 195137 (2014).
[17] P. Delplace, Á. Gómez-León, and G. Platero, Phys. Rev. B 88, 245422 (2013).

[18] S. Koghee, L.-K. Lim, M. O. Goerbig, and C. Morais Smith, Phys. Rev. A 85, 023637 (2012).

[19] M. Benito, A. Gómez-León, V. M. Bastidas, T. Brandes, and G. Platero, Phys. Rev. B 90, 205127 (2014).

[20] M. C. Rechtsman, J. M. Zeuner, Y. Plotnik, Y. Lumer, D. Podolsky, F. Dreisow, S. Nolte, M. Segev, and A. Szameit, Nature 496, 196 (2013).

[21] Y. Wang, H. Steinberg, P. Jarillo-Herrero, and N. Gedik, Science 342, 453 (2013).

[22] N. Fläschner, B. Rem, M. Tarnowski, D. Vogel, D.-S. Lühmann, K. Sengstock, and C. Weitenberg, Science 352, 1091 (2016).

[23] J. Wang and J. Gong, Phys. Rev. A 77, 031405 (2008).

[24] D. Y. H. Ho and J. Gong, Phys. Rev. B 90, 195419 (2014).

[25] M. Lababidi, I. I. Satija, and E. Zhao, Phys. Rev. Lett. 112, 026805 (2014).

[26] Z. Zhou, I. I. Satija, and E. Zhao, Phys. Rev. B 90, 205108 (2014).

[27] K.-H. Ding, L.-K. Lim, G. Su, and Z.-Y. Weng, Phys. Rev. B 97, 035123 (2018).

[28] N. Goldman, J. Dalibard, M. Aidelsburger, and N. R. Cooper, Phys. Rev. A 91, 033632 (2015).

[29] A. Gómez-León and G. Platero, Phys. Rev. Lett. 110, 200403 (2013).

[30] G. Jotzu, M. Messer, R. Desbuquois, M. Lebrat, T. Uehlinger, D. Greif, and T. Esslinger, Nature 515, 237 (2014).

[31] T. Mikami, S. Kitamura, K. Yasuda, N. Tsuji, T. Oka, and H. Aoki, Phys. Rev. B 93, 144307 (2016).

[32] M. S. Rudner, N. H. Lindner, E. Berg, and M. Levin, Phys. Rev. X 3, 031005 (2013).

[33] T. Kitagawa, E. Berg, M. Rudner, and E. Demler, Phys. Rev. B 82, 235114 (2010). 
[34] F. Nathan and M. S. Rudner, New J. Phys. 17, 125014 (2015).

[35] A. Quelle, M. Goerbig, and C. Morais Smith, New J. Phys. 18, 015006 (2016).

[36] M. Wackerl and J. Schliemann, arXiv:1802.01369.

[37] T. P. Grozdanov and M. J. Raković, Phys. Rev. A 38, 1739 (1988).

[38] A. Eckardt and E. Anisimovas, New J. Phys. 17, 093039 (2015).

[39] S. Rahav, I. Gilary, and S. Fishman, Phys. Rev. A 68, 013820 (2003).

[40] A. López, A. Scholz, B. Santos, and J. Schliemann, Phys. Rev. B 91, 125105 (2015).

[41] P. Streda, J. Phys. C 15, L717 (1982).

[42] T. Fukui, Y. Hatsugai, and H. Suzuki, J. Phys. Soc. Jpn. 74, 1674 (2005).

[43] F. D. M. Haldane, Phys. Rev. Lett. 61, 2015 (1988).

[44] T. Oka and H. Aoki, Phys. Rev. B 79, 081406 (2009).

[45] T. Kitagawa, T. Oka, A. Brataas, L. Fu, and E. Demler, Phys. Rev. B 84, 235108 (2011).

[46] W. Beugeling, N. Goldman, and C. Morais Smith, Phys. Rev. B 86, 075118 (2012).
[47] I. Dana, Y. Avron, and J. Zak, J. Phys. C 18, L679 (1985).

[48] E. Kalesaki, C. Delerue, C. Morais Smith, W. Beugeling, G. Allan, and D. Vanmaekelbergh, Phys. Rev. X 4, 011010 (2014).

[49] W. Beugeling, E. Kalesaki, C. Delerue, Y.-M. Niquet, D. Vanmaekelbergh, and C. Morais Smith, Nature Commun. 6, 6316 (2015).

[50] P. Soltan-Panahi, J. Struck, P. Hauke, A. Bick, W. Plenkers, G. Meineke, C. Becker, P. Windpassinger, M. Lewenstein, and K. Sengstock, Nat. Phys. 7, 434 (2011).

[51] N. Goldman, J. Budich, and P. Zoller, Nat. Phys. 12, 639 (2016).

[52] A. Quelle, C. Weitenberg, K. Sengstock, and C. Morais Smith, New J. Phys. 19, 113010 (2017).

[53] Refer to Fig. 7.4 in A. Pfeuffer-Jeschke, Ph.D. thesis, Würzburg University, 2000.

[54] E. G. Novik, A. Pfeuffer-Jeschke, T. Jungwirth, V. Latussek, C. R. Becker, G. Landwehr, H. Buhmann, and L. W. Molenkamp, Phys. Rev. B 72, 035321 (2005). 